\title{
Combating Osteoporosis and Obesity With Green Tea Polyphenols: Molecular Switching of Osteogenesis Versus Adipogenesis During Early Differentiation of Human Adipose Tissue-Derived Stem Cells
}

\author{
Weiguo Lao \\ University of Technology Sydney \\ Yi Tan \\ University of Technology Sydney \\ Michael Johnson \\ University of Technology Sydney \\ Yan Li \\ University of Technology Sydney \\ Yiguang Lin \\ University of Technology Sydney \\ Linda Xiao \\ University of Technology Sydney \\ Xianqin Qu ( $\nabla$ xianqin.qu@uts.edu.au ) \\ University of Technology Sydney
}

Research

Keywords: Human adipose tissue-derived stem cells, Green tea polyphenols, Adipogenesis, PPARY-CEBPA signaling pathway, Osteogenesis, RUNX2-BMP2 pathway

Posted Date: August 5th, 2020

DOI: https://doi.org/10.21203/rs.3.rs-53339/v1

License: (c) (1) This work is licensed under a Creative Commons Attribution 4.0 International License.

Read Full License 


\section{Abstract}

Background: Osteoporosis is a metabolic disease affecting the bone mineral density associated with increased adiposity in the aging population with obesity. The nutrients to control osteoblast and adipocyte differentiation from a common precursor, the pluripotent mesenchymal stem cell (MSC), may

be a promising therapy for osteoporosis. Previously, we have shown that green tea polyphenols (GTP) exert anti-adipogenic effects on preadipocyte proliferation. In the present study, we investigated regulatory effects of GTP on osteogenesis and adipogenesis during early differentiation of human adipose tissue-derived stem cells (hADSCs).

Methods: GTP at concentrations of 1 and $10 \mu \mathrm{g} / \mathrm{ml}$ was incubated with primary hADSCs in presence or absence of pioglitazone $(100 \mu \mathrm{mol})$ during hADSCs differentiation. Adipogenesis of hADSCs was determined by Oil Red $O$ staining and measurement of the cellular triglyceride synthesis in mature adipocyte. Alkaline phosphatase (ALP) assay and the measurement of intracellular calcium were utilized to determine osteoporosis of hADSCs. Immunofluorescence staining and qRT-PCR were employed to detect PPARY-CEBPA regulated adipogenic pathway and the RUNX2-BMP2 mediated osteogenic pathway.

Results: GTP treatment significantly decreased lipid accumulation and the cellular triglyceride synthesis in mature adipocytes and attenuated pioglitazone-induced adipogenesis in a dose-dependent manner. GTP downregulated protein and mRNA expression of Ppary and attenuated pioglitazone-stimulated Cebpa expression in mature adipocytes. Concurrently, measurements of calcium content and ALP activity showed that GTP treatment significantly enhanced hADSCs differentiation into osteocytes compared with the control and pioglitazone-treated cells. Meanwhile, GTP upregulated protein and mRNA expression of RunX2 and Bmp2 compared to the control and GTP at $10 \mu \mathrm{g} / \mathrm{mL}$ significantly attenuated the decreased mRNA expression of Runx2 and Bmp2 by pioglitazone.

Conclusions: The present study demonstrated that GTP possess a greater ability to facilitate osteogenesis and simultaneously inhibit hADSCs differentiation into the adipogenic lineage through upregulating the RUNX2-BMP2 mediated osteogenic pathway and suppressing PPARy-induced signaling of adipogenesis. The findings of this study highlight that GTP may be a therapeutic intervention to combat osteoporosis associated obesity.

\section{Background}

Osteoporosis is a metabolic disease affecting the bone mineral density and a leading cause of bone fracture in the aging population with obesity [1]. The pathophysiology of osteoporosis is multifactorial. Accumulating data have shown that in obesity, the disease may be initialized by abnormal activation of adipogenesis in mesenchymal stem cells (MSCs), which affect MSCs differentiation into osteoblasts, leading to the development of osteoporosis and metabolic disorders [2, 3]. Thus, MSC, including those 
derived from bone marrow (BMSC) and adipose tissue (AD-MSC), have received considerable attention for understanding the pathogenesis of osteoporosis in obesity.

Adipocytes and osteoblasts originate from a common ancestor - pluripotent mesenchymal stem cells and there is an inverse relationship between adipocytes and osteoblasts in the bone marrow [4].

Significantly, inducers of differentiation towards one lineage may inhibit cell differentiation into an alternative lineage. In the aging population with obesity, differentiation to adipocytes dominates over the differentiation to osteoblasts from BMSC and AD-MSC [5]. Thus, the imbalance between osteogenesis and adipogenesis during stem cells differentiation contributes to osteoporosis and tendency for bone fracture in the elderly with obesity. Therefore, the molecular shift of stem cells towards osteogenesis and diminishing adipogenesis may prove important therapeutic approach to osteoporosis [6].

There is growing evidence that nutritional intervention with phytochemicals is effective for controlling obesity and bone loss simultaneously [7]. Epidemiological studies revealed that post-menopausal habitual tea drinkers have higher bone mineral density (BMD) [6] and reduced risk of hip fractures amongst elderly men and women [8-10]. However, the precise mechanism of nutritive polyphenols in maintaining bone health is not fully defined.

Our previous studies showed that GTP exert anti-adipogenic effects on preadipocyte proliferation in 3T3L1 adipocytes [11] and ameliorate metabolic abnormalities and insulin resistance in obese Zucker fatty rats [12]. In the present study we evaluated whether GTP is capable of modulating the differentiation of human adipose tissue-derived stem cells (hADSCs) shifting towards osteogenic lineage and suppressing adipogenesis during early stage of differentiation. Peroxisome proliferator-activated receptor gamma (PPARY), a ligand-activated transcription factor, upregulates MSCs differentiation into adipocytes [13]. In this study, we included PPARY agonist (pioglitazone) as a reference drug to clarify the molecular pathways by which GTP regulate adipogenesis and osteogenesis during the differentiation of hADSCs into mature adipocytes and osteoblasts. Biomarkers and genes involved in the adipogenic and osteogenic pathways were analysed.

\section{Methods}

\section{Green Tea Polyphenols and Pioglitazone}

The green tea polyphenols (GTP) preparation (99\% of total catechins consisting of: $70.9 \%$ epigallocatechin gallate [EGCG], 1.7\% epigallocatechin, 7.4\% epicatechin gallate, and 19.3\% epicatechin) was kindly provided by Zuyi Lushen Kangyuan Co (Zuyi, Guizhou, China). Pioglitazone (Pio) was purchased from Sigma-Aldrich (St. Louis, MO, USA) and was used to induce adipogenesis during hADSC differentiation.

\section{Isolation and culture of human adipose tissue-derived stem cells}


Human adipose tissue-derived stem cells (hADSCs) were kindly provided by Dr Jerran Santos from an existing research project approval under the Macquarie University human research ethics committee (Ref \#: 5201100385). hADSCs were isolated with the method previously described [14]. After isolation, hADSCs were confirmed by CD45 negative and CD90 positive with fluorescein isothiocyanate kits (BD Biosciences, San Jose, CA, USA) using a FC500 flow cytometer (Beckman Coulter, Brea, CA, USA). Primary hADSCs were sub-cultured in Dulbecco modified Eagle medium (DMEM) containing $10 \%$ fetal bovine serum (FBS, Gibco Life Technologies, Auckland, New Zealand) with 1\% penicillin-streptomycin (PS, Gibco Life Technologies, Auckland, New Zealand) to passage 5 to 6 for the following in vitro studies.

\section{hADSCs differentiation and treatment}

To determine the optimal time from the differentiation of primary hADSCs into mature adipocytes, the cells were seeded at a density of $5 \times 10^{3}$ /well in 24-well plate in growth medium (DMEM with $10 \%$ FBS and $1 \% \mathrm{PS}$ ). When the cells were expanded to $70 \%$ confluence (designated as day 0 of differentiation induction), growth medium containing Glutmax/F12 (Gibco Life Technologies, Auckland, New Zealand) with $0.5 \mathrm{mmol}$ isobutyl-methylxanthine (IBMX), $1 \mu \mathrm{mol}$ dexamethasone, $10 \mu \mathrm{mol}$ insulin, $200 \mu \mathrm{mol}$ indomethacin was replaced for adipogenic induction. This adipogenic medium was refreshed every 2 days. The numbers of mature adipocytes was determined by visual inspection of the lipid droplet formation under light microscopy (Olympus, BX51 microscope, Tokyo, Japan) followed by Oil Red O staining (Sigma-Aldrich, St Louis, MO, USA) and triglycerides measurement described previously [11] on day 7, 14, 21 and 28 after induction treatment. Triglyceride contents were increased more than 10 times on day 21 of adipogenic induction.

For determination of optimal time of osteogenic differentiation, growth medium containing $0.1 \mu \mathrm{mol}$ dexamethasone, $50 \mu \mathrm{mol}$ ascorbate-2-phosphate, $10 \mathrm{mmol} \beta$-glycerophosphate was added in cell culture and replaced every 2 days. The mature osteocytes were confirmed by measurement of alkaline phosphatase (ALP) activity and calcium content at each experiment point same as adipogenic induction. Calcium levels reached almost 10 -fold higher than the baseline level in cells on day 14 after osteogenic induction.

According to above time course study, GTP treatment for adiogenesis and osteogenesis was scheduled for 21 days and 14 days after induction, respectively. Briefly, GTP ( $99 \%$ purity) was firstly dissolved at 10 $\mathrm{mg} / \mathrm{ml}$ in sterile distilled water with $0.1 \%$ dimethyl sulfoxide (DMSO) and this preparation was used to achieve the final concentrations at 1 and $10 \mu \mathrm{g} / \mathrm{ml}$ in hADSCs culture in the presence or absence of 100 $\mu \mathrm{mol}$ of pioglitazone. The fresh induction media with treatment reagents was replaced every 2 days.

\section{Determination of lipid accumulation and triglycerides content}

Oil Red O (Sigma-Aldrich, St Louis, MO, USA) staining was used to detect lipid droplets in differentiated adipocytes. Cells were washed with phosphate-buffered saline (PBS) three times and fixed with 10\% formalin at room temperature for 1 hour. After fixation, cells were washed once with PBS and stained with filtered Oil Red $\mathrm{O}$ solution (60\% isopropanol, $40 \%$ water) for 30 minutes. After staining the lipid droplets, 
the Oil Red $\mathrm{O}$ staining solution was removed and the plates were rinsed with water to remove unbound dye, dried and photographed. The stained lipid droplets were viewed with an Olympus microscope (Tokyo, Japan) and images were captured with digital camera (DP70, Tokyo, Japan), then quantitated by using Image-Pro6.2 software (Media Cybernetics, Inc. MD, USA) from 4-5 individual experiments.

Quantitation of cellular triglycerides content was performed on day 21 after differentiation induction and treatment. After washing with PBS, differentiated cells were harvested with $200 \mu \mathrm{L}$ PBS then centrifuged at $1000 \times g$ for 10 minutes. The pellet was dissolved in $1 \mathrm{ml}$ lipid extracting solution (chloroform: methanol $=2: 1$ ). After drying under nitrogen gas, lipids were lysed in $100 \mu \mathrm{l}$ ethanol. The triglycerides concentration in the cell lysates were quantified using the commercial triglyceride assay kit (Wako Pure Chemical Industries, Osaka, Japan) from 5 individual experiments. The concentration of triglycerides was calculated according the standard curve. The cellular content of triglycerides was adjusted based on the quantity of protein.

\section{Alkaline phosphatase assay and determination of intracellular calcium}

The Alkaline phosphatase (ALP) is expressed in the early stages of differentiation and is a marker of osteoblastic phenotype. hADSCs were cultured in osteogenic differentiation media with variety of treatments for 14 days. At the end of treatment, cells were harvested from each well with $0.05 \%$ trypsin$0.53 \mathrm{mmol}$ EDTA and washed with PBS, lysed in $1 \mathrm{ml}$ of $0.2 \%$ Triton X-100 aqueous solution using a Vibra-Cell sonicator (VXC 500 series, Sonic and Materials Inc., CT, USA) for 30 seconds. The sonicates were centrifuged at 10,000 $\times \mathrm{g}$ for 10 minutes and the supernatants were collected for ALP activity assay using a Chemistry Analyzer (Abbott Architect ci16200, Abbott Park, Illinois, USA) according to the manufacturer's instructions.

To detect intracellular calcium content, the harvested cells were dissolved in $200 \mu \mathrm{L}$ of $0.5 \mathrm{M} \mathrm{HCl}$ then vigorously shaken for 16 hours at room temperature then the mixtures were sonicated for 30 seconds followed by centrifugation at $5000 \times \mathrm{g}$ for 10 minutes. The supernatant was collected for calcium assay using Chemistry Analyzer (Abbott Architect ci16200, Abbott Park, Illinois, USA) followed the manual instruction. The calcium contents were calibrated with cells number counted in the each well.

\section{Immunofluorescence staining and quantification}

PPAR $y$ protein expression on adipogenic differentiation and runt-related transcription factor 2 (Runx2) protein expression on osteogenic differentiation were determined with immunofluorescence staining. Primary hADSCs were grown cells $\left(2 \times 10^{4}\right.$ cells/well) were plated on glass coverslips in 24-well plates until $70 \%$ confluence then were maintained for 14 days of osteogenic induction and 21 days of adipogenic induction with variety of treatments. At the end of treatment, cells were washed with cold PBS then fixed in $4 \%$ paraformaldehyde for 20 minutes, permeabilized with PBS-T solution (PBS with $0.06 \%$ Tween 20 and 0.04\% Triton 100) for 5 minutes, blocked with 5\% Bovine Serum Albumin (BSA) for 30 minutes. After blocking, cells were incubated with primary antibody overnight at $4^{\circ} \mathrm{C}$. Primary antibodies and dilutions were: rabbit anti-PPARy (Santa Cruz Biotechnology, Texas, USA 1:100) or mouse anti-Runx2 
monoclonal (Cell Signaling Technology Danvers, MA, USA 1:100) in 2\% BSA-PBS and incubated overnight at $4^{\circ} \mathrm{C}$. $2 \%$ BSA-PBS solution was used for negative control. After incubation with the primary antibody, cells were washed with fresh PBS followed by staining with secondary antibodies: goat anti-rabbit or goat anti-mouse IgG Alexa Fluor 488 (Thermo Fisher Scientific, MA USA 1: 400) for 1 hour at room temperature in dark. After washing with PBS for 3 times, the coverslip contained the cells was mounted on glass slides with SlowFade Diamond Antifade Mountant with Propidium lodide (PI) (Gibco Life Technologies, Auckland, New Zealand) for 2 minutes at room temperature. Images were obtained using a fluorescent microscope (Olympus BX51 and DP70, Tokyo, Japan) and fluorescent intensity of the antibody labelled regions within cells was calculated from the images using Image $\mathrm{J}$ software (National Institutes of Health, Bethesda, MD, USA) from 4 individual experiments.

\section{Total RNA isolation and quantitative real-time PCR}

To further clarify molecular mechanism by which GTP modulate hADSCs differentiation, the gene expression of Ppary, enhancer-binding protein alpha (Cebpa) and the cyclic adenosine monophosphate (cAMP) responsive element binding protein ( $\mathrm{Creb}$ ) which regulate adipogenesis, as well as Runx 2 and bone morphogenetic protein 2 (Bmp2) genes which regulate osteogenesis, were determined by quantitative real-time polymerase chain reaction (qRT-PCR).

Amplification of complementary DNA (cDNA) with primers (Thermo Fisher Scientific, Massachusetts, USA) showing in the Table 1. cDNA was carried out using Stratagene MXPro-Mx3000P (Agilent Technologies, Waldbronn, Germany). qRT-PCR was performed with QuantStudio 6k Flex Real-Time System (Applied Biosystems, Foster City, CA, USA) using SYBR FastStart Universal SYBR Green Master Mixes (Roche, Mannheim, Germany) in accordance with the manufacturer's instruction. The Delta Ct value (Ct [targeted gene] - $\mathrm{Ct}[\mathrm{Actb}]$ ) was used as a measure of relative changes messenger RNA (mRNA) of Cebpa, Ppary, Creb, Runx2 and Bmp2 in GTP treated cells and compared with mRNA levels of positive and negative controls. Each sample was amplified in triplicate, and the expression of Beta-actin (Actb) was used as an internal control for every PCR assay.

\section{Statistical analyses}

Statistical analyses were performed using GraphPad Prism version8.0 (GraphPad Software, San Diego, CA, USA) and all values are expressed as means \pm SE. Differences between groups were examined using one-way ANOVA followed by Tukey's test to determine significant differences between the groups. $P$ value $<.05$ were considered to be statistically significant.

\section{Results}

GTP reduces lipids accumulation and triglyceride synthesis duringthe dfferentiation of hADSCs into adipocytes 
To examine the effects of GTP on adipocyte differentiation, lipid accumulation was determined on day 21 of differentiation cells with 1 and $10 \mu \mathrm{g} / \mathrm{mL}$ of GTP alone or a combination with $100 \mu \mathrm{mol}$ of pioglitazone. Figure 1A showed the intracellular lipid droplets stained by Oil Red $O$ and quantitative results of lipids accumulation (Fig. 1B). Pioglitazone significantly increased $(P<.001)$ the total amount of lipids compared with the control cells. GTP prevented lipid accumulation (both $P<.05$ vs. control cells). Furthermore, GTP attenuated Pio-induced lipid accumulation at dose-dependent manner $(P<.05$ and $P<.01$ vs. pioglitazone alone) during ADSCs differentiation to adipocytes.

In concordance with reduction of total lipid contents, intracellular triglyceride levels were reduced $(P<.05)$ in GTP treated cells compared with the control cells. Pioglitazone stimulated triglyceride production $(P<$ .01 vs. the control) and this was markedly attenuated by the addition of GTP (Fig. 1C).

\section{GTP inhibits the expression of PPARY protein and genes involved in ADSCs towards adipogenic differentiation}

PPARY is a key transcription factor that is upregulating genes expression involved in adipogenesis. To elucidate the mechanism by which GTP inhibits adipogenesis during ADSCs differentiation, PPARY expression was detected with immunofluorescence staining and PPARY agonist (pioglitazone) was used as a reference drug. Figure 2 shows that pioglitazone at $100 \mu \mathrm{mol}$ stimulated expression of PPARY by $24.1 \pm 0.78 \%$ compared to the control $(P<.05)$. GTP treatment at 1 and $10 \mu \mathrm{g} / \mathrm{mL}$ downregulated PPARY expression by $22.1 \pm 0.97 \%(P<.05)$ and $44.3 \pm 1.3 \%(P<.01)$ respectively, when compared with the control. When GTPs were added on pioglitazone, the increased PPARY expression was diminished by 24.3 $\pm 1.03 \%$ with $10 \mu \mathrm{g} / \mathrm{ml}$ of GTP $(P<.05)$, suggesting that GTP prevented pioglitazone-induced PPARY overexpression.

PPARY, CCAAT-enhancer-binding proteins (C/EBPa) and CREB play a crucial role in adipogenic pathway. To define the molecular mechanism by which GTPs negatively regulate hADSCs towards adipocyte differentiation, transcription factors of Ppary, Cebpa and Creb, were analyed with qRT-PCR. Pioglitazone significantly increased the expression of $\operatorname{Ppary}(P<.01)$ and Cebpa $(P<.05)$ but did not significantly affect the mRNA of Creb. GTP at low dose $(1 \mu \mathrm{g} / \mathrm{mL})$ did not affect the expression of these transcription factors but $10 \mu \mathrm{g} / \mathrm{mL}$ of GTP downregulated PparX expression $(P<.05)$ when compared to the control (Fig. 3A). GTP treatment prevented pioglitazone-stimulated mRNA expression of Cebpa (Fig. 3B, $P<.05$ ) and $\mathrm{Creb}$ (Fig. 3C, $P<.05)$.

\section{GTP stimulates mineralization and ALP activity during osteogenic differentiation of hADSCs}

Calcium deposition and ALP activity were used to characterize mineralization and osteogenic differentiation of hADSCs towards mature osteocytes. Figure 4 shows calcium contents and ALP activity in the cells on days 14 after osteogenic induction with variety of treatments. Pioglitazone (Pio, $100 \mu \mathrm{mol}$ ) significantly decreased calcium contents (Fig. 4A, $P<.05$ vs. the control) and inhibited ALP activity by $25.0 \pm 3.4 \%$ (Fig. 4B, $P<.05$ vs. the control). GTP at $10 \mu \mathrm{g} / \mathrm{mL}$ significantly enhanced calcium contents (Fig. 4A, $P<.05$ vs. the control) associated with increased APL activity (Fig. 4B, $P<.05$ ) but GTP at 1 
$\mu \mathrm{g} / \mathrm{mL}$ neither significantly influenced intracellular calcium levels nor changed ALP activity in mature osteogenic cells, suggesting higher dose of GTP is requested to achieve osteogenic effect. Furthermore, GTP treatment at $10 \mu \mathrm{g} / \mathrm{mL}$ partially reversed the pioglitazone-reduced calcium deposition (Fig. 4B, $P<$ .05 vs. Pioglitazone alone) and ALP activity (Fig. 4B, $P<.05$ and $P<.01$, respectively, vs. pioglitazone alone).

\section{GTP induces the expression of Runx2 protein and genes involved in ADSCs towards osteogenic differentiation}

Runx2 is a key protein molecule involved in the osteogenic differentiation process. To elucidate the mechanism by which GTP enhances osteogenic differentiation, as evidenced by increased calcium contents and ALP activity, protein expression of Runx2 was measured with immunofluorescence staining on day 14 after differentiation induction and treatments. Figure 5 shows that pioglitazone slightly decreased Runx2 protein expression, and GTP treatments ( 1 and $10 \mu \mathrm{g} / \mathrm{mL}$ ) significantly enhanced Runx2 expression by $31.1 \pm 1.4 \%(P<.01)$ and $37.2 \pm 2.1 \%(P<.001)$ compared to the control. Adding GTP to pioglitazone slightly increased Runx2 expression compared to pioglitazone treatment alone, however, the enhancement did not achieve statistical significance even at high dose of GTP $(10 \mu \mathrm{g} / \mathrm{mL})$.

To understand the molecular mechanism of GTP enhanced osteogenic differentiation from hADSCs, mRNA expression of Runx2 and Bmp2 were detected with qRT-PCR. Treatment with pioglitazone did not significantly affect the expression of Runx2 and Bmp2 (Fig. 6A \& B). Interestingly, treatment with 10 $\mu \mathrm{g} / \mathrm{mL}$ of GTP significantly increased expression of Runx2 $(P<.05)$ and $B m p 2(P<.01)$ compared to the control (Fig. 6). The high dose of GTP $(10 \mu \mathrm{g} / \mathrm{mL})$ added on pioglitazone also significantly increased Runx2 $(P<.05)$ and $B m p 2(P<.05)$ expression compared to pioglitazone alone.

\section{Discussion}

Accumulating evidence supports a complex relationship between adiposity and osteoporosis in overweight/obese individuals, with an increase of adipogenesis accompanied by decreasing bone formation in obese populations $[15,16]$. Osteoblasts and adipocytes differentiate from a common precursor, the pluripotent mesenchymal stem cells found in bone marrow and adipose tissue have a reciprocal relationship between the adipogenesis and osteogenesis at the early stage of differentiation [17]. Thus, the tightly controlled lineage commitment of MSCs plays a critical role in the maintenance of bone homeostasis and lean body composition. Downregulation of differentiation towards adipogenesis lineage may promote cell differentiation into osteogenesis lineage. In the present study, we evaluated whether GTP is capable of regulating differentiation of primary human adipose-derived stem cells shifting towards osteogenic lineage and preventing adipogenesis. The results showed that GTP prevents lipid accumulation and reduces triglyceride content in ADSCs destined to differentiate in adipocytic cells following exposure to an adipogenic differentiation medium. This finding is consistent with previous reports that EGCG, a major component of GTP, has the ability to suppress adipogenesis through downregulating gene expression in mice MSCs [18]. Human ADSCs used in this study possess a higher 
stem cell population compared to other MSC sources [17] and its pluripotency behaviour and pathophysiology are more correlative to human obesity than MSCs from mice [18]. This study demonstrated that GTP exert an inhibitory effect on hADSCs adipocyte-differentiation. Furthermore, GTP attenuated pioglitazone-stimulated adipogenesis. It is well known that pioglitazone as a PPARy agonist can enhance insulin sensitivity for type 2 diabetes but adversely affects body composition and bone metabolism $[13,19]$. Our previous study has shown that GPT ameliorate metabolic abnormalities and insulin resistance by enhancing insulin signalling in skeletal muscle of Zucker fatty rats [12]. Taken together, adding GTP on pioglitazone may exert synergetic effect for insulin resistance, hyperglycemia and metabolic complications of obesity.

Besides its anti-adipogenic effect, the present study also confirmed that GTP facilitated hADSCs to differentiate into osteoblasts, as evidenced by two measurements. Firstly, GTP treatment enhanced cellular calcium contents, which are an essential substance for mineralization in mature osteocytes. Secondly, ALP activity was higher in the ADSCs culture after 14 days of exposure to osteogenic differentiation medium with GTP treatment. ALP is a specific osteogenic marker in the early stages of osteogenic differentiation from MSCs and its activity plays an important role in osteocyte mineralization. In this study, the PPARy agonist pioglitazone negatively regulated the differentiation of ADSCs into osteocytes, evidenced by reduction of calcium contents and ALP activity in hADSCs culture with $100 \mu \mathrm{mol}$ of pioglitazone. Interestingly, GTP treatment normalized pioglitazone-reduced calcium and ALP activity. An in vivo study by Shen et al. demonstrated that green tea supplementation prevented body weight gain and improved bone microstructure and strength in obese female rats fed with a high-fat diet $[20,21]$. The results of the present study together with findings of animal studies $[12,20,21]$ indicate that the consumption of GTP may simultaneously prevent the fat formation and enhance bone formation. Furthermore, the combination of GTP with PPARY agonist may be an optimal therapy for diabetic patient associated with obesity and osteoporosis.

To gain an insight into mechanism of GTP promoting osteogenesis and inhibiting adipogensis we examined the expression of molecular protein and genes which regulate early stage of hADSCs differentiation. PPARY and the CCAAT-enhancer-binding protein family (C/EBPa, C/EBP,$C / E B P \delta)$, particularly $\mathrm{C} / \mathrm{EBPa}$, and the CAMP regulatory element-binding protein (CREB) have been identified as master regulators that involve in transcriptional control of the early stages of adipocyte-differentiation [22]. Activated PPARy induces the expression of $\mathrm{C} / \mathrm{EBPa}$, which turns on several genes involved in ADSCdifferentiation to mature adipocyte [23]. Immunofluorescence staining and qRT-PCR revealed that pioglitazone stimulates the expression of PPARY and C/ebpa. GTP treatment at $10 \mu \mathrm{g} / \mathrm{mL}$ significantly reduced the expression of PPARY protein and mRNA, indicating an inhibition of Ppary gene and/or a reduction of the transcription from mRNA to PPARy protein with GTP. GTP did not affect the expression of Cebpa and Creb but GTP prevented pioglitazone-induced Cebpa overexpression. These results critically revealed a mechanism of GTP inhibiting the differentiation of hADSCs into adipocytes by suppressing PPAR $y$-CEBP $a$ mediated adipocyte-differentiation. 
Recent studies have demonstrated that an inverse relationship exists in adipogenic and osteogenic lineage commitment and differentiation [24]. In this study, we speculated that GTP switching PPARYmediated adipogenesis differentiation towards osteoblast differentiation might induce biomarkers in controlling osteogenic differentiation such as Runx2 and bone morphogenetic protein (BMP). The result of this study showed pioglitazone negatively regulate osteogenesis through downregulation of Runx2 expression. Runx 2 is a key gene expressed in the early stages of the osteogenic differentiation process [25]. Increased expression of Runx2 and mRNA in GTP-treated cells indicates its osteogenic activity. GTP treatment also increased Bmp2 expression. Runx2 and Bmp2 are the critical molecular switches involved in the osteogenic differentiation of mesenchymal stem cells [26,27]. These results indicate that GTP is capable of promoting osteogenic differentiation through up-regulating the gene expression of Runx 2 and Bmp2, and inhibiting adipogenic differentiation of ADSCs by down-regulating the expression of Ppary.

\section{Conclusions}

Through utilizing hADSCs and PPARy agonist, this study evaluated regulatory effects of GTP on osteogenesis and adipogenesis. The present study demonstrated that GTP possess a greater ability to facilitate osteogenesis and simultaneously inhibit hADSCs differentiation into the adipogenic lineage through upregulating the RUNX2-BMP2 mediated osteogenic pathway and suppressing PPARY-induced signaling of adipogenesis. The findings of this study highlight that GTP may be a therapeutic intervention to combat osteoporosis associated obesity.

\section{Abbreviations}

ADSCs: adipose tissue-derived stem cells

ANOVA: analysis of variance

ALP: alkaline phosphatase enzyme

BMP2: bone morphogenetic protein 2

cDNA: complementary DNA

CEBPA: enhancer-binding protein alpha

CREB: the CAMP responsive element binding protein

FITC: fluorescein isothiocyanate

GTP: green tea polyphenols

hADSCs: human adipose tissue-derived stem cells

mRNA: messenger RNA

Page 10/19 
MSC: mesenchymal stem cell

PBS: phosphate-buffered saline

PCR: polymerase chain reaction

PPARY: peroxisome proliferator-activated receptor gamma

qRT-PCR: quantitative real-time polymerase chain reaction

Runx2: runt-related transcription factor 2

\section{Declarations}

\section{Availability of data and materials}

The datasets and /or analysed during the current study available from the corresponding author on reasonable request.

\section{Ethics approval and consent to participate}

Not applicable.

\section{Consent for publication}

Not applicable.

\section{Competing interests}

The authors declare that they have no competing interests.

\section{Funding}

The present study was supported by the special international collaboration grant (S2011GR0387) from the Ministry of Science and Technology of the People's Republic of China.

\section{Authors' Contributions}

Qu X conceived and designed study; Lao W conducted majority of cell culture and with Tan Y, Li Y (visiting scholar under Qu X's supervision) and Lin Y performed laboratory assays; Xiao L contributed to chemical analysis of GTP; and Johnson M contributed to analysis of immunofluorescence staining images. Qu X wrote the manuscript and Johnson M reviewed and revised the final draft of the manuscript. All authors read and approved the final manuscript.

\section{Acknowledgments}


We are grateful to Zuyi Lushen Kangyuan Co (Meitan, Guizhou, China) for supporting this study by providing green tea polyphenols. The authors would also like to thank Dr Yali Sun's advice for chemical analysis of green tea polyphenols.

\section{Author information}

\section{Affiliations}

1. School of Life Sciences, University of Technology Sydney, NSW 2007, Australia

Weiguo Lao, Yi Tan, Michael Johnson, Yan Li, Yiguang Lin Linda Xiao and Xianqin Qu

2. Sydney Institute of Traditional Chinese Medicine, Dixon St, Haymarket, 2000, NSW, Australia

Yan Li

\section{Corresponding author}

Correspondence to Xianqin Qu

\section{References}

1. Fassio A, Idolazzi L, Rossini M, Gatti D, Adami G, Giollo A, et al. The obesity paradox and osteoporosis. Eat Weight Disord-St. 2018;23:293-302. https://doi.org/doi:10.1007/s40519-0180505-2.

2. Hu L, Yin C, Zhao F, Ali A, Ma J, Qian A. Mesenchymal Stem Cells: Cell Fate Decision to Osteoblast or Adipocyte and Application in Osteoporosis Treatment. Int. J. Mol. Sci.2018;19. https://doi.org/doi:10.3390/ijms19020360.

3. Singh L, Tyagi S, Myers D, Duque G. Good, bad, or ugly: the biological roles of bone marrow fat. Curr Osteoporos Rep. 2018;16:130-7. https://doi.org/doi:10.1007/s11914-018-0427-y.

4. Horowitz MC, Lorenzo JA. The origin of osteoclasts. Curr Opin Rheumatol. 2004;16:464-8.

5. Fazeli PK, Horowitz MC, MacDougald OA, Scheller EL, Rodeheffer MS, Rosen CJ, et al. Marrow fat and bone-new perspectives. J Clin Endocrinol Metab. 2013;98:935-45. https://doi.org/doi:10.1210/jc.2012-3634.

6. Rayalam S, Della-Fera MA, Baile CASynergism. Between Resveratrol and Other Phytochemicals: Implications for Obesity and Osteoporosis. Mol Nutr Food Re. 2011;55:1177-85. https://doi.org/doi:10.1002/mnfr.201000616.

7. Hegarty VM, May HM, Khaw KT. Tea drinking and bone mineral density in older women. Am J Clin Nutr. 2000;71:1003-7.

8. Wu CH, Yang YC, Yao WJ, Lu FH, Wu JS, Chang CJ. Epidemiological evidence of increased bone mineral density in habitual tea drinkers. Arch Intern Med. 2002;162:1001-6. https://doi.org/doi:10.1001/archinte.162.9.1001. 
9. Kanis J, Johnell O, Gullberg B, Allander E, Elffors L, Ranstam J, et al. Risk factors for hip fracture in men from Southern Europe: The MEDOS study. Osteoporosis Int. 1999;9:45-54. https://doi.org/doi:10.1007/s001980050115.

10. Johnell O, Gullberg B, Kanis JA, Allander E, Elffors L, Dequeker J, et al. Risk-Factors For Hip Fracture In European Women - The Medos Study. J Bone Miner Res. 1995;10:1802-15. https://doi.org/doi:10.1002/jbmr.5650101125.

11. Lao W, Tan Y, Jin XL, Xiao L, Kim JJY, Qu X. Comparison of Cytotoxicity and the Anti-Adipogenic Effect of Green Tea Polyphenols with Epigallocatechin-3-Gallate in 3T3-L1 Preadipocytes. Am J Chin Med. 2015;42:1177-90. https://doi.org/doi:10.1142/S0192415X15500676.

12. Cheng J, Tan Y, Zhou J, Xiao L, Johnson M, Qu X. Green tea polyphenols ameliorate metabolic abnormalities and insulin resistance by enhancing insulin signalling in skeletal muscle of Zucker fatty rats. Clin Sci (Lond). 2020;134:1167-80. https://doi.org/doi:10.1042/cs20200107.

13. Derosa G, Maffioli P. Peroxisome proliferator-activated receptor-y (PPAR- $\gamma$ ) agonists on glycemic control, lipid profile and cardiovascular risk. Curr Mol Pharmacol. 2012;5:272-81.

14. Bunnell BA, Flaat M, Gagliardi C, Patel B, Ripoll C. Adipose-derived stem cells: isolation, expansion and differentiation. Methods. 2008; 45:115 - 20. https://doi.org/doi:10.1016/j.ymeth.2008.03.006.

15. Takada I, Kouzmenko AP, Kato S. Molecular switching of osteoblastogenesis versus adipogenesis: implications for targeted therapies. Expert Opin Ther. 2009;13:593-603. https://doi.org/doi:10.1517/14728220902915310.

16. Bunnell BA, Flaat M, Gagliardi C, Patel B, Ripoll C. Adipose-derived stem cells: isolation, expansion and differentiation. Methods. 2008; 45:115 - 20. https://doi.org/doi:10.1016/j.ymeth.2008.03.006.

17. Zuk PA, Zhu M, Ashjian P, De Ugarte DA, Huang JI, Mizuno H, et al. Human adipose tissue is a source of multipotent stem cells. Mol Biol Cell. 2002;13:4279-95. https://doi.org/doi:10.1091/mbc.e02-020105.

18. Chani B, Puri V, Chander Sobti R, Puri S. Epigallocatechin Gallate Inhibits Mouse Mesenchymal Stem Cell Differentiation to Adipogenic Lineage. J Stem Cells Regen Med. 2016;12:16-24.

19. de Souza CJ, Eckhardt M, Gagen K, Dong M, Chen W, Laurent D, et al. Effects of Pioglitazone on Adipose Tissue Remodeling Within the Setting of Obesity and Insulin Resistance. Diabetes. 2001;50:1863. https://doi.org/doi:10.2337/diabetes.50.8.1863.

20. Shen CL, Yeh JK, Samathanam C, Cao JJ, Stoecker BJ, Dagda RY, et al. Green tea polyphenols attenuate deterioration of bone microarchitecture in female rats with systemic chronic inflammation. Osteoporos Int. 2011;22:327-37. https://doi.org/doi:10.1007/s00198-010-1209-2.

21. Shen CL, Han J, Wang S, Chung E, Chyu MC, Cao JJ. Green tea supplementation benefits body composition and improves bone properties in obese female rats fed with high-fat diet and caloric restricted diet. Nutr Res. 2015;35:1095-105. https://doi.org/doi:10.1016/j.nutres.2015.09.014.

22. Zhang JW, Klemm DJ, Vinson C, Lane MD. Role of CREB in transcriptional regulation of CCAAT/enhancer-binding protein beta gene during adipogenesis. J Biol Chem. 2004;279:4471-8. doi:10.1074/jbc.M311327200. 
23. Wu Z, Rosen ED, Brun R, Hauser S, Adelmant G, Troy AE, et al. Cross-regulation of C/EBP alpha and PPAR gamma controls the transcriptional pathway of adipogenesis and insulin sensitivity. Mol Cell. 1999;3(00):151-8. https://doi.org/doi:10.1016/s1097-2765. 80306-8.

24. Haakonsson AK, Madsen MS, Nielsen R, Sandelin A, Mandrup S. Acute Genome-Wide Effects of Rosiglitazone on PPAR gamma Transcriptional Networks in Adipocytes. J Mol Endocrinol. 2013;27:1536-49. https://doi.org/doi:10.1210/me.2013-1080.

25. Bruderer M, Richards RG, Alini M, Stoddart MJ. Role and Regulation Of Runx2 In Osteogenesis. Eur Cells Mater. 2014; 28:269 - 86. https://doi.org/doi:10.22203/eCM.v028a19.

26. Chava S, Chennakesavulu S, Gayatri MB, Reddy ABM. A novel phosphorylation by AMP-activated kinase regulates RUNX2 from ubiquitination in osteogenesis over adipogenesis. Cell Death Dis. 2018; 9. https://doi.org doi: 10.1038/s41419-018-0791-7.

27. Yodthong T, Kedjarune-Leggat U, Smythe C, Wititsuwannakul R, Pitakpornpreecha T. I-Quebrachitol Promotes the Proliferation, Differentiation, and Mineralization of MC3T3-E1 Cells: Involvement of the

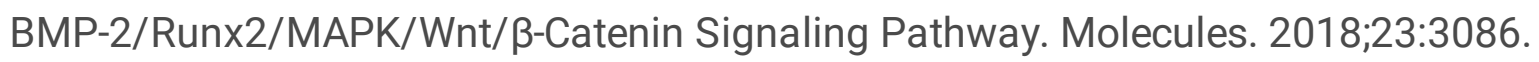
https://doi.org/doi:10.3390/molecules23123086.

\section{Tables}

Table 1

Primer sequences used in real-time PCR

\begin{tabular}{|lll|}
\hline Primer Symbol* & Forward primer & Reverse primer \\
\hline Cebpa & 5'-TATAGGCTGGGCTTCCCCTT-3' & 5'-AGCTTTCTGGTGTGACTCGG-3' \\
\hline Ppar甘 & 5'-CCGTGGCCGCAGATTTGA-3' & 5'-AGATCCACGGAGCTGATCCC-3' \\
Creb & 5'-TTCAAGCCCAGCCACAGATT-3' & 5'-AGTTGAAATCTGAACTGTTTGGAC-3' \\
Runx2 & 5'-CACCGAGACCAACAGAGTCA-3' & 5'-TGGTGTCACTGTGCTGAAGA-3' \\
Bmp2 & 5'-TTTCAATGGACGTGTCCCCG-3' & 5'-AGCAGCAACGCTAGAAGACA-3' \\
Actb & 5'-CTCACCATGGATGATGATATCGC-3' & 5'-AGGAATCCTTCTGACCCATGC-3' \\
\hline
\end{tabular}

\section{Figures}



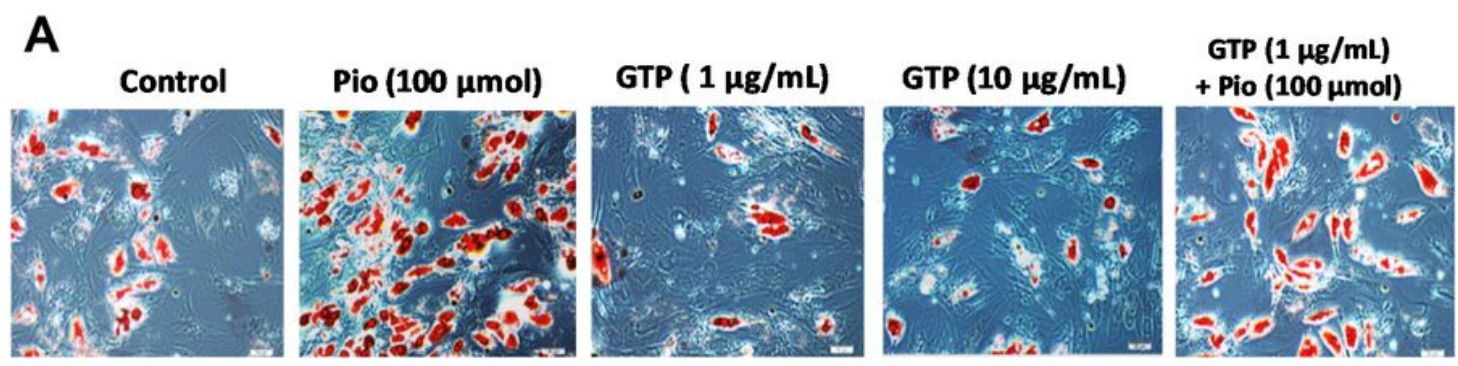

GTP $(10 \mu \mathrm{g} / \mathrm{mL})$

+ Pio $(100 \mu \mathrm{mol})$
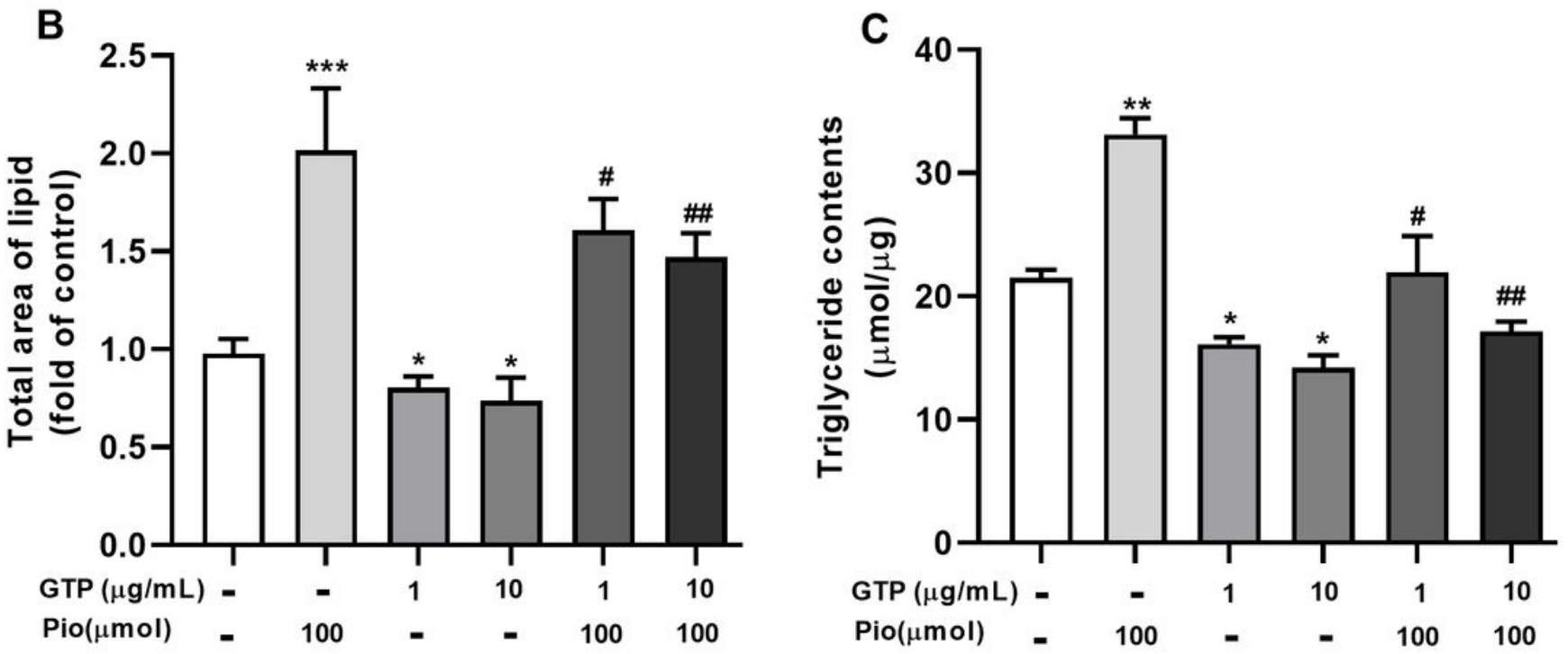

\section{Figure 1}

Green tea polyphenols (GTP) decrease lipids accumulation and triglyceride synthesis during hADSCs adipogenic differentiation. A. Image of the lipid droplets with Oil Red O (ORO) staining and examined by a light microscopy at 100x, objective magnification; B. The quantitative data of total area occupied by lipid droplets stained by ORO; $\mathrm{C}$. Triglycerides content was determined by measuring the absorbance at 490 $\mathrm{nm}$. Data are presented as means $\pm \mathrm{SE}\left(\mathrm{n}=5\right.$ per group). ${ }^{*} \mathrm{P}<.05,{ }^{\star}{ }^{\mathrm{P}}<.01$ and ${ }^{*} \star * \mathrm{P}<.001 \mathrm{GTP}$ and pioglitazone vs. the control; \#P $<.05$ and \#\#P $<.01$ GTP plus pioglitazone (Pio) vs. Pio alone. 

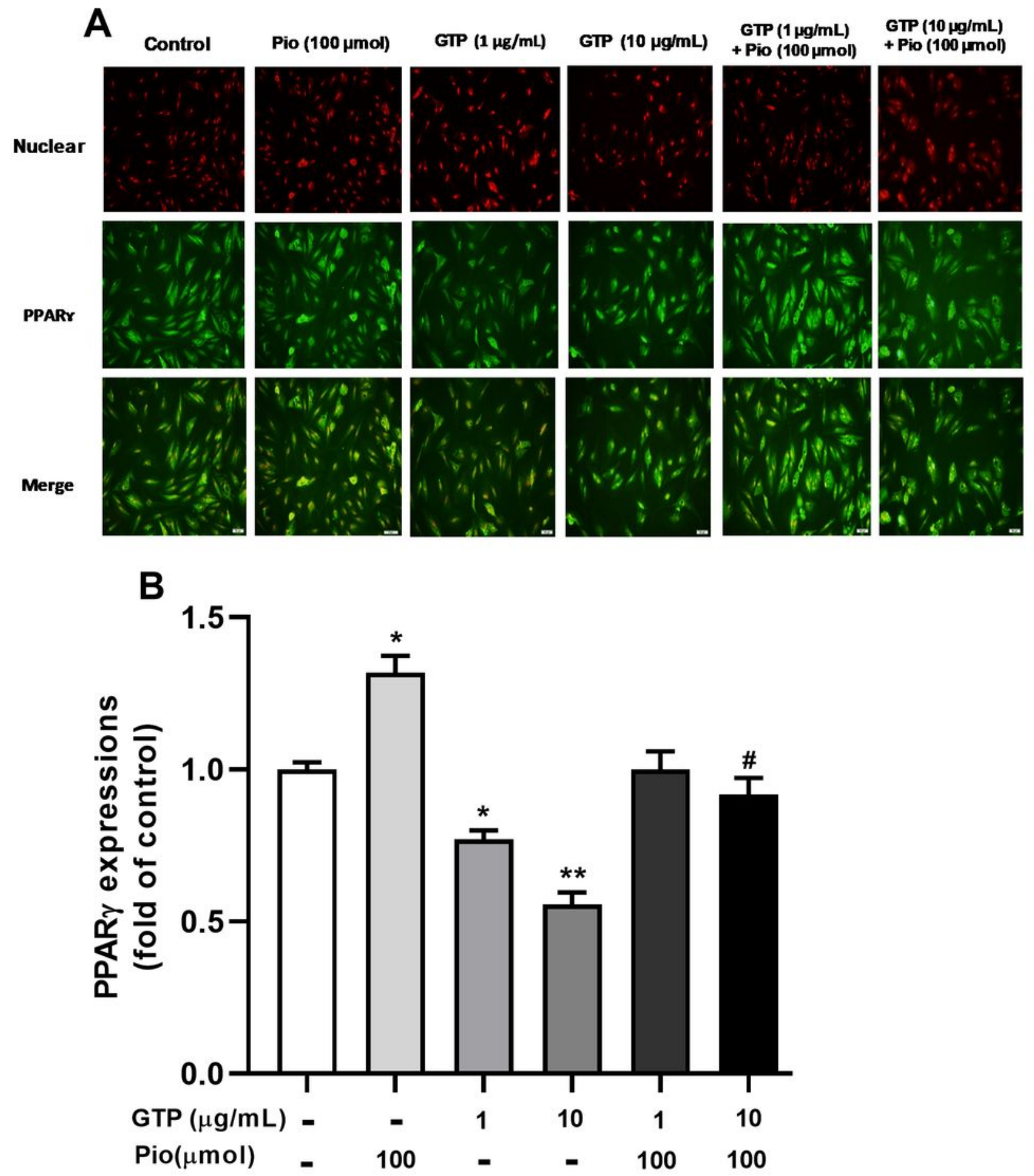

Figure 2

Green tea polyphenols (GTP) inhibit PPARy expression during hADSCs adipogenic differentiation. Images of immunofluorescence staining (A) depicted a marked elevation in the fluorescent intensity of PPARY antibodies conjugated to FITC stained as green colour in the cells with pioglitazone (Pio, $100 \mu \mathrm{mol}$ ) treatment and reduction in the fluorescent intensity of PPAR $\triangle$ antibodies conjugated to FITC in the cellstreated with GTP (1 and $10 \mu \mathrm{g} / \mathrm{mL})$. Quantitative data (B) are expressed as means \pm SE. ${ }^{*} \mathrm{P}<.05$ and **P 
$<.01 \mathrm{GTP}$ and pioglitazone treatments vs. the control. \#P $<.05$ GTP plus pioglitazone vs. pioglitazone alone.
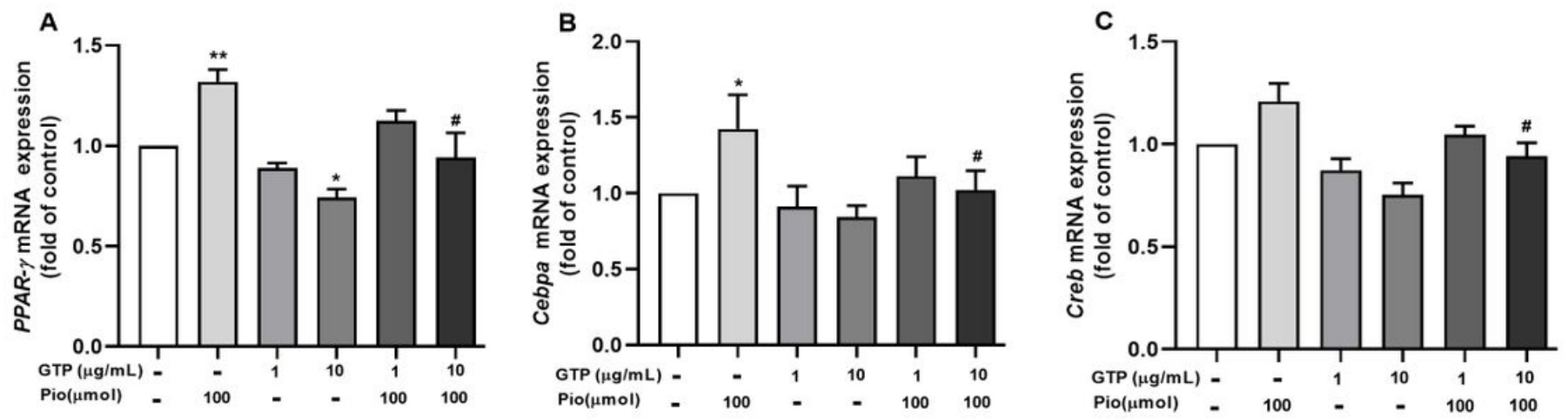

Figure 3

Effects of green tea polyphenols (GTP) on mRNA expression of Ppary Cebpa and Creb. Gene expression of Ppary (A), Cebpa (B) and Creb (C) were detected by qRT-PCR in the cell cultures with DMEM (control), pioglitazone (Pio, $100 \mu \mathrm{mol})$, GTP (1 and $10 \mu \mathrm{g} / \mathrm{mL}$ ) and GTP plus Pio for 21 days. Data from 4 separate experiments are expressed as means \pm SE. ${ }^{*} \mathrm{P}<.05$ and ${ }^{* *} \mathrm{P}<.01 \mathrm{GTP}$ and pioglitazone treatments $\mathrm{vs}$. the control. \#P $<.05$ GTP plus pioglitazone vs. pioglitazone alone.
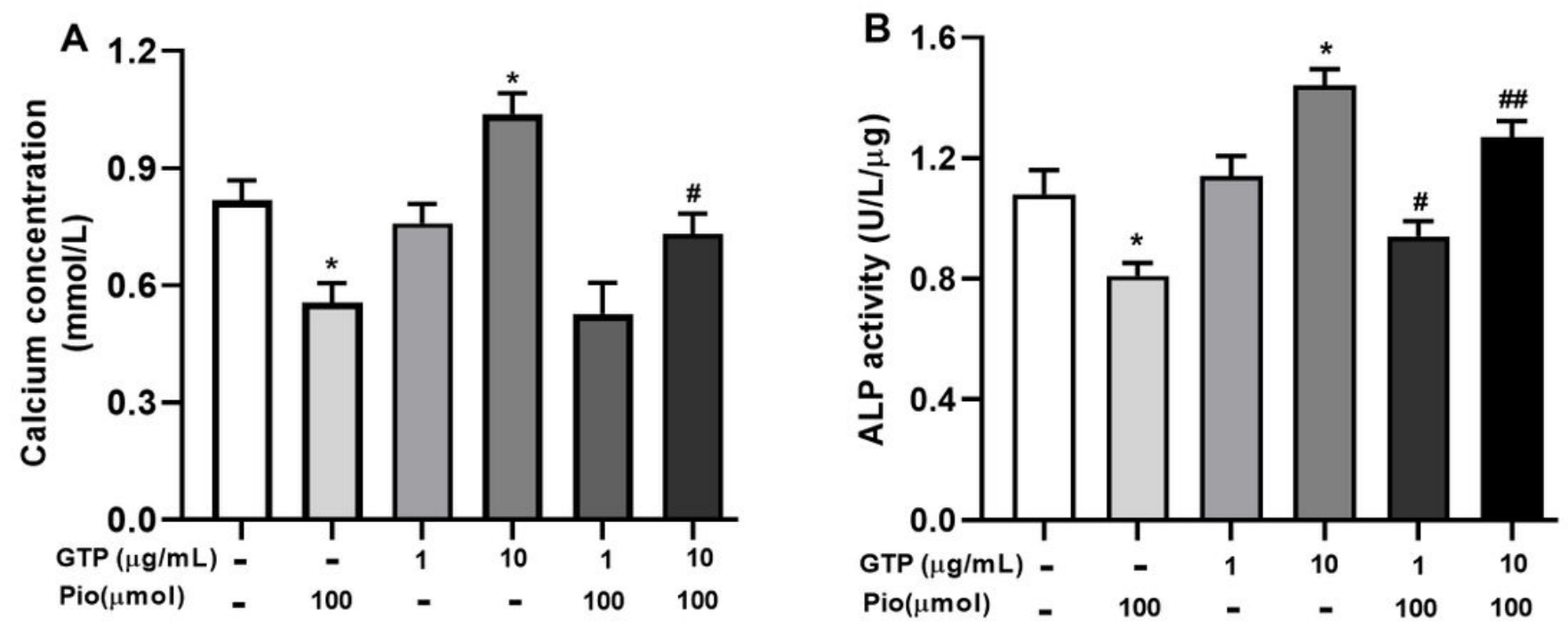

Figure 4

Green tea polyphenols (GTP) decrease calcium contents and ALP activity in mature osteocytes. The quantitative assay of calcium level (A) and ALP activity (B) was performed by an automatic biochemistry instrument (ARCHITECT, Japan). Data from 4 separate experiments are presented as means $\pm \mathrm{SE}$. $* \mathrm{P}<$ 
.05 pioglitazone and GTP vs. the control; \#P < .05 and \#\#P <.01 GTP plus pioglitazone vs. pioglitazone treatment alone.
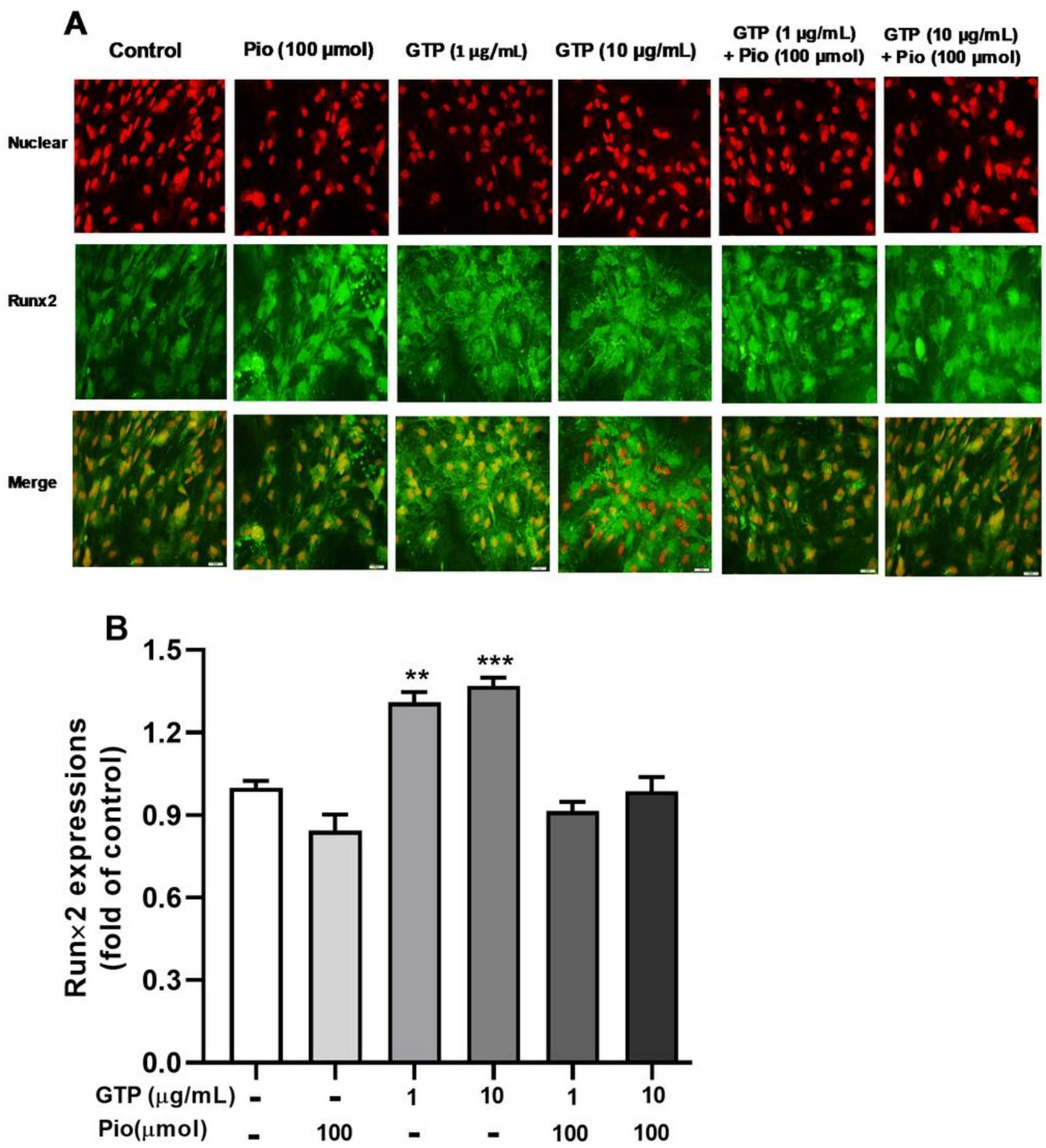

Figure 5

Green tea polyphenols (GTP) enhance Runx2 expression during hADSCs towards oestrogenic differentiation. Images of immunofluorescence staining (A) showed Runx2 protein contents stained as green colour in the cells treated with pioglitazone (Pio, $100 \mu \mathrm{mol}$ ), 1 and $10 \mu \mathrm{g} / \mathrm{mL}$ of GTP and the 

as means \pm SE. ${ }^{\star *} \mathrm{P}<.01$ and ${ }^{\star *} \mathrm{P}<.001 \mathrm{GTP}$ treatments vs. the control.
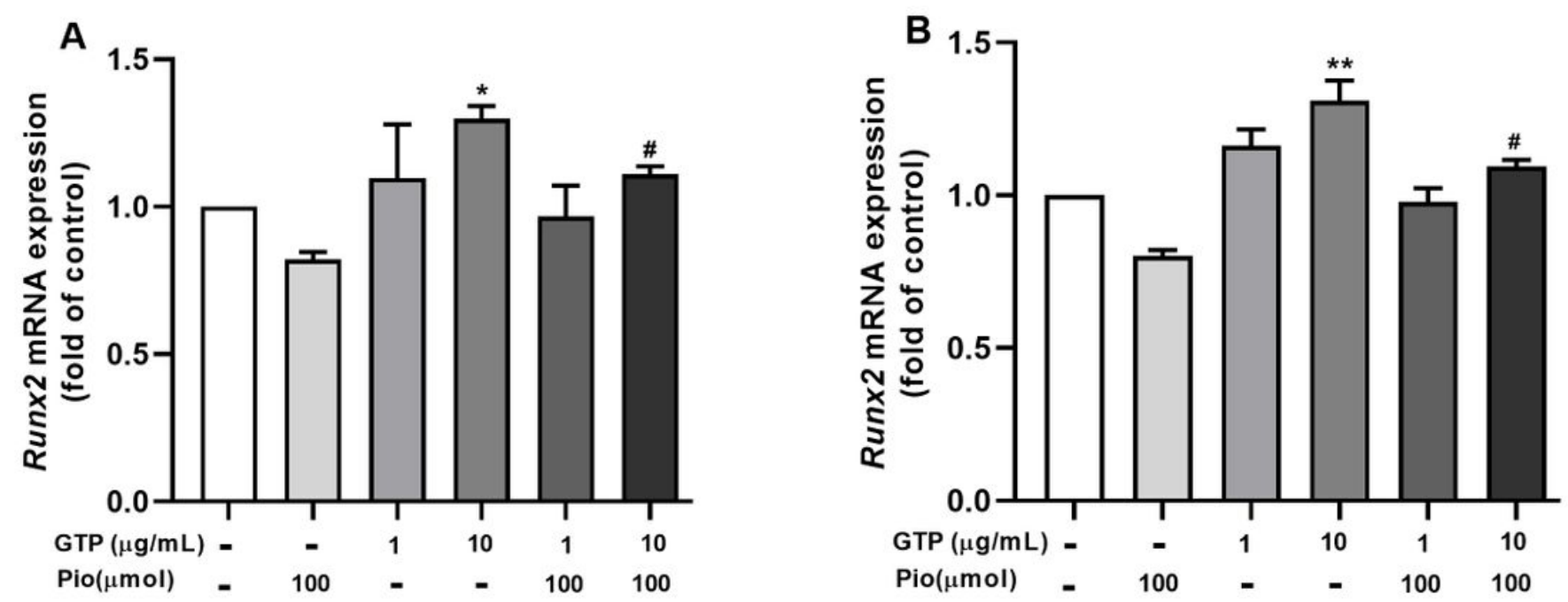

Figure 6

Green tea polyphenols (GTP) upregulate mRNA expression of Runx2 and Bmp2 in mature osteocytes. Gene expression of Runx2 (A) and Bmp2 (B) analysed with qRT-PCR in the cell underwent 14 days of oestrogenic induction with variety of treatments. Data from 4 separate experiments are expressed as means $\pm \mathrm{SE} .{ }^{*} \mathrm{P}<.05$ and ${ }^{* *} \mathrm{P}<.01 \mathrm{GTP}$ treatments vs. the control. \#P $<.05$ GTP plus pioglitazone vs. pioglitazone alone.

\section{Supplementary Files}

This is a list of supplementary files associated with this preprint. Click to download.

- Figurelegend.docx 